\title{
Seguimiento farmacoterapéutico de pacientes chilenos con infección por VIH/SIDA hospitalizados en un centro de alta complejidad durante un período de un año
}

\author{
Fernando Bernal, Patricia Vásquez, Lorena Rodríguez, Alejandra Gavrilovics y Michel Serri
}

Hospital San Juan de Dios Santiago.

Unidad de Infectología.

Conflicto de interés: ninguno.

Fuente financiamiento: ninguna.

Recibido: 3 de marzo de 2014 Aceptado: 25 de noviembre 2014

Correspondencia a: Fernando Bernal Ortiz fernando.bortiz@gmail.com

\author{
Pharmacotherapy follow-up of Chilean inpatients with HIV/AIDS in a high \\ complexity hospital during one year
}

Background: Pharmaceutical follow-up consists on the detection, prevention, and resolution of drug-related problems (DRP) in a continuous, systematized, and documented way. DRP is a health problem connected to pharmacotherapy, which can interfere with the expected health outcomes in the patient. Aim: To make a pharmacotherapeutic surveillance in HIV/AIDS inpatients attended at "Hospital San Juan de Dios" throughout a year prospective study (January to December 2012). Results: $88.6 \%(93 / 105)$ of patients were in stage AIDS with a median of CD4+ lymphocytes of 29 cells $/ \mathrm{mm}^{3}$ and a viral load of 107,000 RNA copies $/ \mathrm{mL}$. The $16.2 \%$ were admitted to a intensive care unit ICU. 296 DRP (DRP 2.8 per patient) were detected, 72\% of DRP (n; 213) were associated with treatment safety, 94 were adverse reactions, 19 required dose adjustments, and 6 interactions had a negative clinical impact; $23.3 \%$ (n: 69) experimented the necessity to add or remove a drug and $4.7 \%$ (n: 14) presented problems associated with effectiveness. A statistically significant relation was observed when associating the number of DRP with admittance to the ICU and the use of more than six drugs. Conclusion: The results allow the conclusion that inpatients present drug-related problems mainly related to toxicity, becoming risk factors for the development of them admittance to ICU, and concomitant use of more than 6 drugs.

Key words: Pharmacosurveillance; pharmacotherapeutic follow-up; drug-related problems.

Palabras clave: Farmacovigilancia, seguimiento farmacoterapéutico, problemas relacionados a medicamentos.

\section{Introducción}

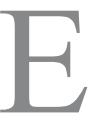
1 seguimiento farmacoterapéutico (SFT) consiste en la detección, prevención y resolución de los problemas relacionados con los medicamentos (PRM), en forma continuada, sistematizada y documentada. Un PRM es un problema de salud vinculado con la farmacoterapia, que interfiere o puede interferir con los resultados de salud esperados en el paciente. Una de las funciones que cumple el químico farmacéutico en los equipos multidisciplinarios es promover el uso adecuado de los medicamentos.

El SFT aborda de manera global todos los problemas de salud y los medicamentos que utiliza el paciente, centrándose en la valoración de la necesidad, efectividad y seguridad de la farmacoterapia. En este sentido, los pacientes con problemas de salud como es la infección por VIH/SIDA, constituyen un grupo con una mayor probabilidad de presentar PRM.

La clasificación de los PRM se fundamenta en los tres requisitos básicos de una farmacoterapia; que sea necesaria, efectiva y segura. A partir de las cuales el Tercer
Consenso de Granada establece seis tipos o categorías de PRM, tal como se muestra a continuación:

\begin{tabular}{|c|c|}
\hline Necesidad & $\begin{array}{l}\text { Problema de salud no tratado } \\
\text { El paciente sufre un problema de salud asociado a no } \\
\text { recibir una medicación que necesita }\end{array}$ \\
\hline & $\begin{array}{l}\text { Efecto de medicamento innecesario } \\
\text { El paciente sufre un problema de salud asociado a } \\
\text { recibir un medicamento que no necesita }\end{array}$ \\
\hline Efectividad & $\begin{array}{l}\text { Inefectividad no cuantitativa } \\
\text { El paciente sufre un problema de salud asociado a } \\
\text { una inefectividad no cuantitativa de la medicación } \\
\text { Inefectividad cuantitativa } \\
\text { El paciente sufre un problema de salud asociado a } \\
\text { una inefectividad cuantitativa de la medicación }\end{array}$ \\
\hline Seguridad & $\begin{array}{l}\text { Inseguridad no cuantitativa } \\
\text { El paciente sufre un problema de salud asociado a } \\
\text { una inseguridad no cuantitativa de un medicamento } \\
\text { Inseguridad cuantitativa } \\
\text { El paciente sufre un problema de salud asociado a } \\
\text { una inseguridad cuantitativa de un medicamento }\end{array}$ \\
\hline
\end{tabular}


Del mismo modo, estos PRM pueden ser clasificados como reales o potenciales. Un PRM real es aquel que se ha manifestado clínicamente en el paciente, produciendo un problema de salud; un PRM potencial es aquel que, sin haberse manifestado todavía, tiene la posibilidad de presentarse provocando el problema de salud.

\section{Objetivos}

- Realizar un seguimiento farmacoterapéutico (SFT) a pacientes con infección por VIH/SIDA hospitalizados.

- Caracterizar demográficamente a los pacientes ingresados, de acuerdo a género, edad, etapa clínica, y unidad de ingreso al momento de la hospitalización.

- Detectar, identificar y clasificar los problemas relacionados al uso de medicamentos y sus intervenciones en pacientes con infección por VIH/SIDA hospitalizados.

\section{Métodos}

Se realizó un estudio prospectivo de 365 días (enero a diciembre 2012) para la detección de PRM en pacientes con infección por VIH/SIDA internados en el Hospital San Juan de Dios (Santiago, Chile). Se elaboró una ficha de seguimiento y se realizaron al menos dos visitas semanales, una de las cuales era en compañía del equipo de Infectología. Durante estas visitas se corroboraron datos de los fármacos, la dosificación de cada uno de ellos y posibles reacciones adversas e interacciones.

El trabajo no precisó ser presentado al Comité de Ética del hospital por deberse a funciones propias del farmacéutico clínico dentro del establecimiento.

\section{Métodos de reclutamiento}

Se incluyeron a todos los pacientes con infección por VIH/SIDA confirmados y/o con clara sospecha clínica (serología para $\mathrm{VIH}$ reactiva con detección de antígeno p24, más presencia de una infección oportunista), ingresados en las unidades de Medicina Agudo, Neurología o unidad de Paciente Critico (UPC) del Hospital San Juan de Dios, excluyendo del trabajo a pacientes cuyo egreso fuera menor a siete días (fallecimiento o alta precoz), lo que impedía contar con al menos dos evaluaciones en conjunto con el equipo de infectología.

\section{Análisis estadístico}

Con los datos obtenidos se realizó un análisis estadístico univariado y multivariado para determinar la correlación entre diversas variables y el número de PRM a través de un modelo quasipoisson, usando el método de máxima verosimilitud restringida con el software R 3.1.0

\section{Resulltados}

Ciento once pacientes fueron ingresados al estudio, de los cuales, seis pacientes fueron retirados al no cumplir con los criterios de inclusión (por egreso menor a 7 días desde el ingreso). El 88,6\% (93/105) de los pacientes ingresados se encontraba en etapa SIDA, con una mediana de linfocitos TCD4+ de 29 céls $/ \mathrm{mm}^{3}$ y una carga viral de 107.000 copias ARN/mL. El 16,2\% (n: 17) ingresó a UPC. La caracterización demográfica y clínica de los pacientes se incluye en la Tabla 1.

El 47\% (n: 49) de los ingresos se enmarcaron en el contexto del debut de su enfermedad. Dentro de los motivos de hospitalización, 64,7\% correspondían a infecciones oportunistas, siendo la más frecuente la neumonía por Pneumocystis jiroveci (PCP), seguido del diagnostico de tuberculosis (no meníngea) y toxoplasmosis cerebral $(28,5 \%, 18,8 \%$ y $15,5 \%$, respectivamente). El $20 \%$ de los pacientes ingresados falleció durante el trascurso de su hospitalización.

Durante el estudio fueron detectados 296 problemas asociados a la farmacoterapia, lo que equivale a 2,8 PRM por paciente. De éstos, $69,9 \%$ correspondieron a PRM reales y $30,1 \%$ correspondieron a PRM potenciales. (Figura 1).

El $72 \%$ de los PRM (n: 213) fueron problemas asociados a seguridad, ya fuera toxicidad o eventos adversos, 23,3\% (n: 69) estuvieron relacionados con la necesidad de incorporar o eliminar algún fármaco (PRM de necesidad) y 4,7 \% (n: 14) debido a problemas asociados a efectividad (PRM de efectividad) (Figura 1).

De los pacientes ingresados, $77,5 \%$ presentó algún problema de farmacoterapia durante el estudio; $32,6 \%$ de ellos (n: 28) presentaron un PRM, 14\% (n: 12) presentaron dos PRM, 18,6\% (n: 16) presentaron tres PRM y 34,9 \%30 (n: 30$)$ restante presentaron cuatro o más PRM.

No se observaron diferencias estadísticamente signi-

Tabla 1. Características demográfica e inmunológicas pacientes en seguimiento. Estudio de farmacovigilancia en pacientes con infección por VIH/SIDA. Hospital San Juan de Dios, enero-diciembre de 2012 (n: 105)

\begin{tabular}{lcc|} 
Variable & $\mathbf{n}$ & $\%$ \\
Edad (promedio \pm DE) & & $40 \pm 10,2$ \\
Género & & \\
$\quad$ Masculino & 83 & 79 \\
$\quad$ Femenino & 22 & 21 \\
Unidad de ingreso & & \\
$\quad$ UPC & 17 & 16,2 \\
$\quad$ Neurología & 21 & 20 \\
$\quad$ Medicina agudo & 67 & 63,8 \\
Etapa clínica & & 11,4 \\
$\quad$ VIH (A1,A2, B2) & 12 & 88,6 \\
$\quad$ SIDA (A3,B3,C1,C2,C3) & 93 & Promedio \\
Inmuno-virológico & Mediana & 97 \\
$\quad$ Recuento de linfocitos TCD4+ céls/mm & 29 & 447.616 \\
$\quad$ Carga viral copias ARN/mL & 107.000 & \\
\hline
\end{tabular}




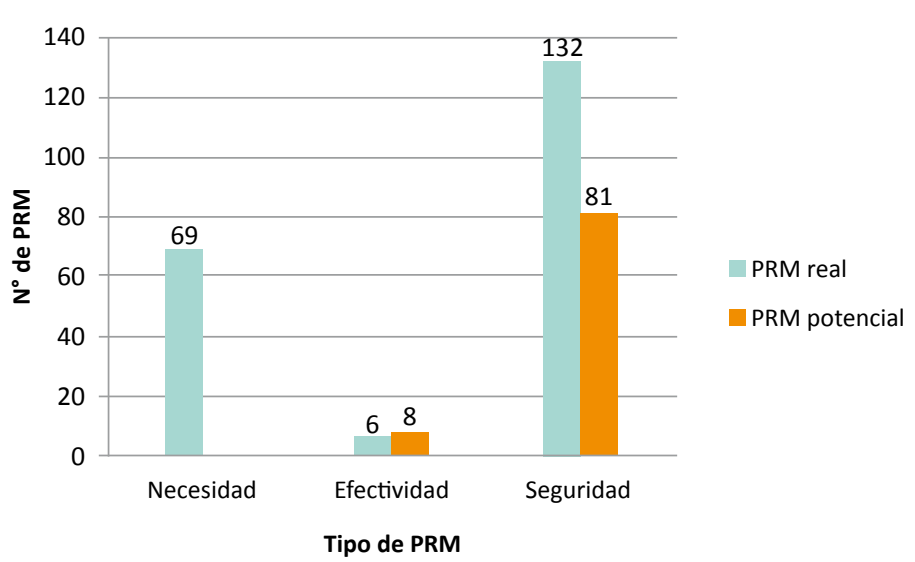

Figura 1. Distribución tipos de PRM encontrados (n: 269) ficativas al comparar los grupos según distribución por sexo.

Al describir los PRM detectados, pudimos observar que:

- De los problemas asociado a la seguridad (PRM seguridad): 49,3\% (n: 105) involucró reacciones adversas, de las cuales 94 se objetivaron en el paciente, 39,4\% (n: 84$)$ de estos problemas se asociaron a interacciones farmacológica y sólo 7,14\% (n: 6) de ellas tuvo repercusión clínica negativa. Se realizaron 19 ajuste de dosis ya fuese por alteración de la función hepática, renal o según peso de los fármacos involucrados; tres de estos ajustes fueron a causa de interacciones.

En las Tablas 2 y 3 se describen con detalle las RAM reales detectadas y las interacciones con repercusión clínica negativa.

Tabla 2. Descripción de reacciones adversas a medicamentos reales observadas.

Estudio de farmacovigilancia en pacientes con infección por VIH/SIDA. Hospital San Juan de Dios, enero-diciembre de 2012. (n: 94)

\begin{tabular}{|c|c|c|c|c|}
\hline Fármaco causal & RAM & n RAM por fármaco sospechoso & Frecuencia & $\%$ del total de RAM \\
\hline Ganciclovir & $\begin{array}{l}\text { Leucopenia } \\
\text { Anemia } \\
\text { Hipokalemia }\end{array}$ & 16 & $\begin{array}{c}13 \\
2 \\
1\end{array}$ & $\begin{array}{c}13,8 \\
2,1 \\
1,1\end{array}$ \\
\hline Zidovudina & $\begin{array}{l}\text { Anemia } \\
\text { Leucopenia } \\
\text { Intolerancia gástrica (cefalea, náuseas) } \\
\text { Melanoniquia longitudinal }\end{array}$ & 14 & $\begin{array}{l}7 \\
3 \\
3 \\
1\end{array}$ & $\begin{array}{l}7,4 \\
3,2 \\
3,2 \\
1,1\end{array}$ \\
\hline Lopinavir/ritonavir & Intolerancia gastrointestinal (diarrea) & 12 & 12 & 12,8 \\
\hline Cotrimoxazol & $\begin{array}{l}\text { Exantema cutáneo } \\
\text { Intolerancia gástrica } \\
\text { Neutropenia } \\
\text { Hiperkalemia }\end{array}$ & 10 & $\begin{array}{l}4 \\
3 \\
2 \\
1\end{array}$ & $\begin{array}{l}4,3 \\
3,2 \\
2,1 \\
1,1\end{array}$ \\
\hline Anfotericina B desoxicolato & Hipokalemia & 6 & 6 & 6,4 \\
\hline Furosemida & Hipokalemia & 6 & 6 & 6,4 \\
\hline Rifampicina & $\begin{array}{l}\text { Colestasia } \\
\text { Aumento transaminasas (GOT, GPT) } \\
\text { Exantema } \\
\text { Pigmentación rojiza en sudor u orina }\end{array}$ & 6 & $\begin{array}{l}2 \\
2 \\
1 \\
1\end{array}$ & $\begin{array}{l}2,1 \\
2,1 \\
1,1 \\
1,1\end{array}$ \\
\hline Atazanavir & Hiperbilirrubinemia indirecta & 5 & 5 & 5,3 \\
\hline Fluconazol & Aumento transaminasas (GOT, GPT) & 4 & 4 & 4,3 \\
\hline Efavirenz & Trastorno SNC (delirio, sueños vívidos) & 3 & 3 & 3,2 \\
\hline Morfina & $\begin{array}{l}\text { Vómitos, náuseas } \\
\text { Prurito }\end{array}$ & 2 & $\begin{array}{l}1 \\
1\end{array}$ & $\begin{array}{l}1,1 \\
1,1\end{array}$ \\
\hline Heparina & Trombocitopenia & 2 & 2 & 2,1 \\
\hline Metronidazol & Diarrea & 1 & 1 & 1,1 \\
\hline TARV & Aumento transaminasas (GOT, GPT) & 1 & 1 & 1,1 \\
\hline Prednisona & Hiperglicemia & 1 & 1 & 1,1 \\
\hline Sulfato Ferroso & Intolerancia gástrica & 1 & 1 & 1,1 \\
\hline Ritonavir & Exantema & 1 & 1 & 1,1 \\
\hline Claritromicina & Disgeusia & 1 & 1 & 1,1 \\
\hline Isoniazida & Colestasia & 1 & 1 & 1,1 \\
\hline Imipenem & Nefrotoxicidad & 1 & 1 & 1,1 \\
\hline
\end{tabular}


Tabla 3. Descripción de interacciones farmacológicas con repercusión clínica negativa.

Estudio de farmacovigilancia en pacientes con infección por VIH/SIDA. Hospital San Juan de Dios, enero-diciembre de 2012. (n: 6)

$\begin{array}{ll}\text { Fármacos involucrados } & \text { Efecto potencial } \\ \text { Amiodarona/Nerapamilo } & \begin{array}{l}\text { Aumento del riesgo de bradicardia, bloqueo atrioventricular y/o } \\ \text { arresto sinusal por inhibición del metabolismo de fármacos involu- } \\ \text { crado a través del CIP3A4 }\end{array} \\ \begin{array}{ll}\text { Zidovudina/dapsona } \\ \text { Letronidazol }\end{array} & \begin{array}{l}\text { Dapsona puede inhibir el metabolismo hepático de zidovudina. } \\ \text { Incremento del riesgo de toxicidad hematológica aditiva }\end{array} \\ \text { Norepinefrina/Linezolid } & \begin{array}{l}\text { Por inhibición del metabolismo de acetaldehído; el uso concomi- } \\ \text { tante de metronizadol y soluciones con alto porcentaje de alcohol, } \\ \text { puede resultar en reacciones de tipo disulfiram (dolor abdominal, } \\ \text { vómitos, cefaleas o flushing) o muerte súbita } \\ \text { Linezolid es un inhibidor débil de la enzima monoamino-oxidasa, } \\ \text { que participa en el metabolismo de neurotransmisores, la admi- } \\ \text { nistración conjunta con este tipo de fármacos puede ocasionar } \\ \text { aumento de presión arterial y síndrome serotoninérgico }\end{array} \\ \text { Fluconazol/Rifampicina } & \begin{array}{l}\text { Por inducción enzimática del ClP3A4, el uso concomitante de } \\ \text { fluconazol y rifampicina, puede disminuir las concentraciones plas- } \\ \text { máticas de fluconazol y disminuir la actividad antifúngica }\end{array}\end{array}$

Fármacos involucrados

Aumento del riesgo de bradicardia, bloqueo atrioventricular y/o arresto sinusal por inhibición del metabolismo de fármacos involucrado a través del CIP3A4

Dapsona puede inhibir el metabolismo hepático de zidovudina.

Por inhibición del metabolismo de acetaldehído; el uso concomi(dolor abdominal,

*Recomendaciones basadas en los análisis de interacciones hechos a través de base de datos Micromedex $₫$ Healthcare Series.

\section{Recomendación ${ }^{29, *}$}

Interacción mayor que justifica evitar el uso concomitante de amiodarona con bloqueadores de canales de calcio en pacientes con síndrome del nodo sinusal o bloqueo atrioventricular parcial

Moderada interacción que puede justificar dosis de zidovudina. Monitorizar toxicidad de zidovudina

El consumo de alcohol en cualquier forma (bebidas, preparados o formas farmacéuticas con alto porcentaje de alcohol) está contraindicado durante el tratamiento con metronidazol, incluso 3 días después de la suspensión del antibiótico

Interacción moderada que justifica monitorizar valores de presión arterial y síntomas serotoninérgicos.

Moderada Interacción que justifica monitorizar la actividad antifúngica y considerar aumento de dosis de fluconazol si se usa concomitantemente con rifampicina.
- Sobre los problemas asociados a la efectividad (PRM efectividad) de los tratamientos, éstos involucraron principalmente interacciones de carácter farmacocinético, es decir, afectarían las concentraciones plasmáticas de algunos de los fármacos involucrados en $64,3 \%$ (n: 9) y en $35,7 \%$ (n: 5) por necesidad de cambio de formulación dada la indicación médica de instalar una sonda nasogástrica (Tabla 4).

- De los problemas asociados a la necesidad (PRM necesidad), destaca 44,9\% (n: 31) generado por necesidad de inicio precoz de tratamiento anti-retroviral, 15,9\% (n: 11) inicio de profilaxis para PCP y 11,6\% (n: 8) por necesidad de transfusión de eritrocitos por anemia, entre otros.

Finalmente, se observó que existe una relación entre el número de PRM y ciertas variables:

- Unidad de ingreso: Los pacientes que ingresan a una unidad de medicina agudo poseen $63 \%$ menos riesgo de presentar un PRM adicional comparado con aquellos que ingresan a una UPC según el análisis univariado y $54 \%$ menos riesgo en el análisis multivariado.

- Número de fármacos: En el analisis multivariado los pacientes que reciben entre cuatro y seis fármacos concomitantes tienen 2,7 veces más riesgo de presentar un PRM adicional en comparación a aquellos que reciben como máximo tres fármacos, riesgo que aumenta hasta 5,5 veces si éste recibe más de seis fármacos en forma concomitante.
- Edad y sexo: No se logró establecer diferencia estadística significativa en los modelos.

\section{Discusión}

En la caracterización demográfica predominó el sexo masculino (79\%), lo que concuerda con lo informado en la literatura médica ${ }^{2}$. Por otra parte, la mediana de linfocitos T CD4+ observada, de 29 céls $/ \mathrm{mm}^{3}$, presenta diferencia significativa con lo informado en la Cohorte Chilena de SIDA del año 2007; al respecto, los pacientes de nuestro estudio representan a aquellos que son diagnosticados en un ambiente hospitalario y no el universo (ambulatorios + hospitalizados) como es informado en la evaluación nacional ${ }^{2}$. Así, 47\% correspondían a pacientes que se diagnosticaron en el debut de su enfermedad. Estos datos sugieren que a pesar de todos los esfuerzos por difusión y promoción de la prevención y diagnóstico precoz del VIH/SIDA, sigue realizándose el diagnostico en etapas tardías de la enfermedad.

Mayores y mejores estrategias para evitar "oportunidades perdidas" en el diagnóstico, tal como: ofrecimiento de la prueba de VIH en pacientes con o sin sospecha y concientizar tanto a la comunidad médica como a la población general de las características clínicasepidemiológica del VIH/SIDA, deben ser consideradas como posibles políticas públicas para reducir el ingreso 
Tabla 4. Interacciones farmacológicas potenciales detectadas.

Estudio de farmacovigilancia en pacientes con infección por VIH/SIDA. Hospital San Juan de Dios, enero-diciembre de 2012. (n: 9)

\begin{abstract}
Fármacos involucrados
\end{abstract}
Ertapenem/Midazolam

Lopinavir-ritonavir/sertralina

Levotiroxina/alimentos

Lopinavir-ritonavir/Fenitoína

Lopinavir-ritonavir/

Carbamazepina

Atazanavir/omeprazol

\section{Efecto potencial}

Interacción de carácter farmacéutico. Físicamente incompatibles. Su mezcla a través de una vía endovenosa única resulta en la aparición de un precipitado blanco denso.

lopinavir-ritonavir puede aumentar los niveles plasmáticos de sertralina por inhibición de su metabolismo a nivel del CIP3A4

La presencia de alimentos en el estómago reduce la absorción de levotiroxina.

Por inducción del CIP3A fenitoína reduce en un 33\% la Cmax y en un $46 \%$ el AUC de lopinavir-ritonavir. Además de reducir las concentraciones en el estado de equilibro de fenitoína.

Por inhibición del CIP3A y efecto bidireccional, la coadministración de estos fármacos puede resultar en la disminución de los niveles plasmáticos de lopinavir-ritonavir e incremento de los niveles de carbamazepina aumentando el riesgo de toxicidad.
Recomendación ${ }^{(27)}(*)$

n

No recomendada. Ambos medicamentos deben ser administrados por separado, sin mezclar en vía o suero de dilución.

Potencial interacción que puede justificar ajuste de dosis. Monitorizar toxicidad de sertralina

No recomendada. Se sugiere administración sin alimentos para evitar efecto de quelación.

Potencial interacción que sugiere monitorizar respuesta a tratamiento antirretroviral $y / 0$ preferir anticonvulsivantes sin efecto inductor enzimático.

Interacción mayor que sugiere recomendar evitar administración concomitante de ambos fármacos, junto con controlar los niveles plasmáticos de carbamazepina 3 a 5 días de iniciada la terapia antiretroviral.

La solubilidad de atazanavir se reduce a medida que aumenta el Ph gástrico. La administración de omeprazol reduce en $70 \%$ los niveles plasmáticos de atazanavir
No recomendada. Se sugiere reemplazar omeprazol por anti-H2 (ranitidina) y administrarlo 2 horas antes de atazanavir o separar lo más posible la administración de omeprazol en pacientes vírgenes a tratamiento antirretroviral.

*Recomendaciones basadas en los análisis de interacciones hechos a través de base de datos Micromedex® Healthcare Series.

de pacientes con inmunosupresión intensa e infecciones oportunistas.

Se detectaron 296 PRM, de los cuales 132 correspondieron a PRM reales relacionados con la seguridad. Este valor es alto y puede ser causa de que la mayor cantidad de PRM en pacientes con infección por VIH, es atribuida a RAM e interacciones ${ }^{3-7}$. Sin embargo, no es posible comparar los datos entregados por este estudio con otras fuentes, ya que no existen reportes referentes a la incidencia de PRM en paciente seropositivos para VIH. Los datos entregados en la literatura médica indican el porcentaje de cada subtipo de PRM, o bien los analizan por separado, es decir, los estudian como reacciones adversas, interacciones, errores de prescripción, etc.; no los engloban en un concepto común. Una revisión reciente observó un bajo número de artículos científicos vinculados a estudios prospectivos y observacionales, dejando de manifiesto la necesidad de poner mayor atención en la identificación y determinación con precisión de los problemas asociados a los medicamentos 8 .

Los PRM son frecuentes en pacientes bajo tratamiento anti-retroviral, encontrándose en el ámbito ambulatorio problemas asociados a la adherencia al tratamiento y seguridad, vinculados al tratamiento anti-retroviral (interacciones, impacto metabólico) ${ }^{9-10}$. Esto difiere en el ambiente hospitalario donde los problemas se centran principalmente en la seguridad del tratamiento, tales como la tolerancia y seguridad de la terapia anti- retroviral, la complejidad añadida por otros tratamientos concomitantes y las interacciones entre fármacos ${ }^{6-7,11}$. Algunos estudios reportan una alta prevalencia de las interacciones farmacológicas como principal causa de errores de medicación y PRM en pacientes con infección por $\mathrm{VIH}$, con porcentajes que oscilan entre $21,7^{7}$ y $80 \% 0^{8,12}$.

Con referencia a los efectos adversos pesquisados en este estudio, la reacción adversa más frecuente fue hematológica. Del total de RAM hematológicas, 13,8 y $3,2 \%$ de los pacientes presentaron leucopenia con el uso de ganciclovir parenteral y zidovudina, respectivamente, y algún grado de anemia en 2,1 y 7,4\% de ellos. Si bien la anemia es una complicación común y seria de la infección por VIH y de su tratamiento, sus causas suelen ser multifactoriales (inmunológicas, virológicas y características propias de los pacientes); su incidencia en pacientes infectados por VIH al momento de su admisión en el centro hospitalario varía de 63 a $95 \%{ }^{13-15}$. Otros estudios han publicado una incidencia de anemia asociada a AZT que oscilan entre 10 y $35 \%{ }^{17-20}$, en nuestro estudio hemos encontrado una baja incidencia de anemia asociada a este fármaco; sin embargo, la mayoría de los pacientes que ingresa a hospitalizarse, como los estudios señalan, lo hacen con una anemia de base que repercute en preferir como base del tratamiento anti-retroviral análogos nucleosídicos con menor riesgo de toxicidad hematológica como abacavir o tenofovir. 
En este estudio no se detectaron errores de medicación en la prescripción de anti-retrovirales. La presencia de subespecialistas y la asignación de la prescripción de estos fármacos a infectólogos, reduce la incidencia de una inadecuada prescripción. Nuestro centro de estudio es un hospital de alta complejidad que cuenta con dotación de subespecialistas para el manejo y control de pacientes infectados por VIH/SIDA. No obstante, en otros países y en algunas otras ciudades chilenas la prescripción de esta familia terapéutica recae en manos de médicos no subespecialistas, encontrándose en variadas publicaciones que los errores de medicación suelen ser frecuentes en pacientes hospitalizados -hasta 35\% de errores-, entre ellos el más común es la omisión (65\%); otros son dosis insuficiente, sobredosis, duplicación de terapia, programación incorrecta y/o terapia inadecua$\mathrm{da}^{21-22}$. Si bien se señala que los errores son subsanados rápidamente durante las primeras $48 \mathrm{~h}$ de la admisión, se requieren intervenciones para proteger a los pacientes y prevenir complicaciones derivadas de los errores de medicación asociados a los anti-retrovirales ${ }^{23}$.

Con respecto a las interacciones detectadas, algunos estudios les asignan una gran importancia debido a que reportan una alta prevalencia de ellas, como también por la gravedad que revisten ${ }^{24-27}$. Si bien en nuestro estudio hemos observado una baja prevalencia de interacciones potenciales y reales, la integración de un químico farmacéutico al equipo médico tratante y en rondas clínicas, puede optimizar la farmacoterapia permitiendo disminuir y/o evitar la aparición de éstas, antes de la prescripción de los fármacos.

En nuestro análisis, un tercio de los PRM detectados corresponden al grupo de pacientes admitidos en una UPC; creemos que es importante ahondar más en futuros trabajos en este grupo de pacientes donde el número de eventos adversos, interacciones y problemas de efectividad puede resultar mayores, debido a las alteraciones de las propiedades farmacocinéticas y/o farmacodinámicas de los fármacos sumando a esto, las características fisiopatológicas de los pacientes críticos.

Tanto en el análisis univariado como en el multivariado, el aumentar el número de fármacos utilizados y el ingreso a UPC presentaron una asociación con significancia estadística a favor del aumento de riesgo de presentar un PRM adicional. Una mayor prescripción de fármacos podría aumentar la complejidad de la posología, incrementando la probabilidad de desarrollar una interacción farmacológica y/o aumentar la complejidad del tratamiento de una patología concomitante (tuberculosis, meningitis criptocóccica). Por otro lado, la complejidad de la unidad de ingreso juega un rol importante. Una UPC atiende pacientes que pueden cursar descompensaciones orgánicas e hidroelectrolíticas importantes, además de requerir el uso de procedimientos invasores y fármacos vasoactivos que aumentan la complejidad del manejo farmacoterapeutico de dicho paciente.

\section{Conclusiones}

Los pacientes con infección por VIH/SIDA internados presentan problemas asociados al uso de medicamentos, ya sea por necesidad, toxicidad o eficacia de los tratamiento recibidos.

Al relacionar el número de PRM con ciertas variables, se observaron factores que aumentan el riesgo de presentar un PRM. En este análisis, los pacientes que poseían entre cuatro y seis o más de seis prescripciones tuvieron hasta 5,5 veces más riesgo de desarrollar un PRM adicional, en comparación a aquellos que poseían como máximo tres fármacos. Por otra parte, el ingresar a una unidad de agudos o neurológica redujo en aproximadamente $50 \%$ (0,46 y 0,54 RTi, respectivamente) el riesgo de presentar un PRM adicional en comparación a una UPC.

La integración del químico farmacéutico al equipo de trabajo en labores clínicas, permite optimizar la farmacoterapia, identificando en forma precoz los problemas relacionados a medicamentos.

\section{Resumen}

Introducción: El seguimiento farmacoterapéutico, consiste en la detección, prevención y resolución de los problemas relacionados con los medicamentos (PRM), en forma continua, sistematizada y documentada. Un PRM es un problema de salud vinculado con la farmacoterapia, que interfiere o puede interferir con los resultados de salud esperados en el paciente. Objetivo y Métodos: Realizar un seguimiento farmacoterapéutico a pacientes hospitalizados con infección por VIH/SIDA del Hospital San Juan de Dios, a través de un estudio prospectivo de un año (enero a diciembre 2012). Resultados: El 88,6\% (93/105) de los pacientes se encontraba en etapa SIDA, con una mediana de linfocitos TCD4+ de 29 céls $/ \mathrm{mm}^{3}$ y una carga viral de 107.000 copias ARN/ $\mathrm{mL}$. El 16,2\% de los pacientes ingresó a una unidad de paciente crítico. Se detectaron 296 PRM (2,8 PRM por paciente); el 72\% de los PRM (n: 213) estuvieron asociados a seguridad del tratamiento, objetivándose 94 reacciones adversas, 19 eventos con necesidad de ajustes de dosis y seis interacciones con repercusión clínica negativa; 23,3\% (n: 69) a la necesidad de incorporar o eliminar algún fármaco y 4,7 \% (n: 14) a problemas asociados a efectividad. Se observó una relación estadísticamente significativa al asociar el $\mathrm{N}^{\circ}$ de PRM con 
la unidad de pacientes críticos y uso de más de seis fármacos. Conclusión: Los resultados permiten concluir que los pacientes hospitalizados presentan problemas relacionados al uso de medicamentos, principalmente asociado a toxicidad, encontrándose como factores de riesgo para el desarrollo de éstos la permanencia en una unidad de paciente crítico y el uso concomitante de más de seis fármacos.

\section{Referencias bibliográficas}

1.- Tercer Consenso de Granada sobre PRM. Ars Pharm 2007; 48 (1): 5-17.

2.- Ministerio de Salud (MINSAL) Comisión Nacional del SIDA y Grupo SIDA Chile Evaluación de impacto del programa de acceso expandido a tratamiento antirretroviral. Cohorte Chilena de SIDA, diciembre 2007.

3.- Panel on Antiretroviral Guidelines for Adults and Adolescents. Guidelines for the use of antiretroviral agents in HIV-1-infected adults and adolescents. Department of Health and Human Services. 2014; 1-285.

4.- Cipolle R, Strand L, Morley P. Drug therapy problems. Pharmaceutical Care Practice. The Clinician's Guide. 2 th Edition. USA, Mc Graw-Hill, 2004. p 171-200.

5.- Parya Saberi, Betty J Dong, et al. The impact of HIV clinical pharmacists on HIV treatment outcomes: a systematic review. Patient Prefer Adherence 2012; 6: 297-322.

6.- Heelon M, Skiest D, et al. Effect of a clinical pharmacist's interventions on duration of antiretroviral-related errors in hospitalized patients. Am J Health Syst Pharm 2007; 64 (19): 2064-8.

7.- American Society of Hospital Pharmacy. 2003. ASHP statement on the pharmacist's role in the care of patients with HIV infection. Am J Health- Syst Pharm 2003; 60: 1998-2003.

8.- Carcelero E, Tuset M, Martin M, et al. Evaluation of antiretroviral-related errors and interventions by the clinical pharmacist in hospitalized HIV-infected patients. HIV Medicine 2011, 12: 494-9.

9.- Piscitelli S C, Gallicano K D. Interactions among drugs for HIV and opportunistic infections. N Engl J Med 2001; 344 (13): 984-96.

10.- Marzolini C, Back D, Weber R, Furrer H, Cavassini M, Calmy A, et al. Ageing with HIV: medication use and risk for potential drug-drug interactions. J Antimicrob Chemother 2011; 66 (9): 2107-11.

11.- Manias E. Detection of medication-related problems in hospital practice: a review . Br J Clin Pharmacol 2013; 76 (1): 7-20.

12.- Mok S, Minson Q. Drug-related problems in hospitalized patients with HIV infection. Am J Health-Syst Pharm. 2008; 65: 55-9.

13.- Grimes R, L Lal, Lewis S T. Frequency of medical history items, drug interactions, and lifestyle characteristics that may interfere with antiretroviral medications. HIV Clin Trials 2002; 3 (2): 161-7.

14.- Semba R, Shah N, Klein R, Mayer K, Schuman P, Vlahov. Prevalence and cumulative incidence of an risk factors for anemia in a multicenter cohort study of human immunodeficiency virus-infected anduninfec-ted women. Clin Infect Dis 2002; 34: 260-6.

15.- Mir N, Costello C, Luckit J, Lindley R: HIV-disease and bone marrow changes: A study of 60 cases. Eur J Haematol 1989; 42: 339.

16.- Frontiera M, Myers A M. Peripheral blood and bone marrow abnormalities in the acquired immunodeficiency syndrome. West J Med 1987; 147: 157.

17.- Zon L I, Arkin C, Groopman J. Haematological manifestations of the human immunodeficiency virus (HIV). Br J Haematol 1987; 66: 251.

18.- Camacho J, Poveda F, Zamorano A F, Valencia M E, VÁsquez J J, Arnalich F. Serum erythropoietin levels in patients with advanced human immunodeficiency virus infection. Br J Haematol 1992; 82: 608.

19.- Horsburgh C R. Mycobacterium avium complex infection in the acquired immunodeficiency syndrome. N Engl J Med 1991; 324: 1332.

20.- Naides S J, Howard E J, Swack N S, True C A, Stapleton J T. Parvovirus B 19 infection in human immunodeficiency virus type-1-infected persons failing or intolerant to zidovudine therapy. J Infect Dis 1993; 168: 101.

21- Richman D D, Fischl M A, Grieco M H, Gottleib M S, Voldering P A, et al. The toxicity of azidothymidine (AZT) in the treatment of patients with AIDS and AIDS-related complex. A double-blinded, placebo controlled trial. N Engl L Med 1987; 317: 192.

22.- Rao N, Patel V, Grigoriu A, Kaushik P, Brizuela M. Antiretroviral therapy prescribing in hospitalized HIV clinic patients. HIV Med 2012; 13 (6): 367-71.

23-. Rastegar DA, Knight A M, Monolakis J S. Antiretroviral medication errors among hospitalized patients with HIV infection. Clin Infect Dis 2006; 43 (7): 933-8.

24.- Yehia B R, Mehta J M, Ciuffetelli D, Moore R D, Pham P A, et al. Antiretroviral medication errors remain high but are quickly corrected among hospitalized HIVinfected adults. Clin Infect Dis 2012; 55 (4): 593-9.

25.- Grimes R L, Lewis S T. Frequency of medical history items, drug interactions, an lifestyle characteristics that may interfere with antiretroviral medications. HIV Clin Trials 2002; 3: 161-7.

26.- Kashuba A. Drug-drug interactions and the pharmacotherapy of HIV infection. Top HIV Med 2005; 13: 64-9.

27.- Lee B, Safrin S. Interactions and toxicities of drugs used in patients with AIDS. Clin Infect Dis 1992; 14: 773-9

28.- Jiménez V. Calidad Fármaco-terapéutica, Universidad de Valencia, Editorial Valencia 2006; pag. 620-3.

29.- Micromedex ${ }^{\circledR}$ Healthcare Series, Drug-Interaction - Thomson Micromedex, Greenwood Village, Colorado (Visitada en Noviembre 2014) link: http://www. micromedexsolutions.com/ 